\title{
Making the World's Skin Crawl: Dermatologic Implications of COVID-19
}

\author{
Kathleen M. Coerdt, BS; Amor Khachemoune, MD
}

\section{PRACTICE POINTS}

- Clinicians should be aware of the skin manifestations of coronavirus disease 19 , especially when triaging patients.

- Health care providers may develop skin diseases from wearing the extensive personal protective equipment required during the current health crisis.

- Coronavirus disease 19 has had a substantial financial impact on dermatologists, and telemedicine may be a potential solution.

$\sim_{1}^{1}$ oronaviruses (CoVs) are among the most common causes of the common cold but also can lead to severe respiratory disease. ${ }^{1}$ In recent years, CoVs have been responsible for outbreaks of severe acute respiratory syndrome (SARS) and Middle East respiratory syndrome (MERS), caused by SARS-CoV and MERS-CoV, respectively. Severe acute respiratory syndrome emerged from China in 2002, and MERS started in Saudi Arabia in 2012. In December 2019, several cases of unexplained pneumonia were reported in Wuhan, China. ${ }^{1}$ A novel $\mathrm{CoV}-\mathrm{SARS}-\mathrm{CoV}-2$ - was isolated in these patients and is now known to cause coronavirus disease 19 (COVID-19). ${ }^{1}$ Coronavirus disease 19 can cause acute respiratory distress and multiorgan failure. ${ }^{1,2}$ It spread quickly throughout the world and was declared a pandemic by the World Health Organization on March 11, 2020. According to the Johns Hopkins University Coronavirus Resource Center (https://coronavirus.jhu.edu/map.html), there were approximately 14,500 COVID-19 cases diagnosed worldwide on February 1, 2020; by May 22, 2020, there were more than 5,159,600 cases. Thus, heightened measures for infection prevention and control were put in place around the globe in an attempt to slow the spread of disease. ${ }^{1}$

In this article, we describe the dermatologic implications of COVID-19, including the clinical manifestations of the disease, risk reduction techniques for patients and providers, personal protective equipment-associated adverse reactions, and the financial impact on dermatologists.

\section{Clinical Manifestations}

At the start of the COVID-19 outbreak, little was known about the skin manifestations of the disease. Providers speculated that COVID-19 could have nonspecific skin findings similar to many other viral illnesses. ${ }^{3,4}$ Research throughout the pandemic has found many cutaneous manifestations of the disease. ${ }^{3-6}$ A case report from Thailand described a patient who presented with petechiae in addition to fever and thrombocytopenia, which led to an initial misdiagnosis of Dengue fever; however, when the patient began having respiratory symptoms, the diagnosis of COVID-19 was discovered. ${ }^{5}$ Furthermore, a study from Italy $(\mathrm{N}=88)$ showed dermatologic findings in $20.4 \%(18 / 88)$ of patients, including erythematous rash (77.8\% [14/18]), widespread urticaria (16.7\% [3/18]), and chickenpoxlike vesicles $(5.6 \%$ [1/18]). A recent study from Spain $(\mathrm{N}=375)$ found 5 cutaneous patterns associated with COVID-19: pseudochilblain-acral areas of erythema with vesicles and/or pustules-lesions (19\%), vesicular eruptions (9\%), urticarial lesions (19\%), maculopapular eruptions (47\%), and livedoid/necrotic lesions (6\%). ${ }^{6}$ Pseudochilblain lesions appeared in younger patients, occurred later in the disease course, and were associated with less severe disease. Vesicular lesions often were found in middle-aged patients prior to the onset of other COVID-19 symptoms, and they were associated with intermediate disease severity. Urticarial and maculopapular lesions typically paralleled other COVID-19 symptoms in timing and were associated with more severe disease. Likewise, livedoid and necrotic lesions were associated with more severe disease; they occurred more frequently in older patients. ${ }^{6}$ Clinicians at Cleveland Clinic found similar cutaneous lesions in COVID-19 patients, including morbilliform rashes, acral purpura resembling perniosis, and

Ms. Coerdt is from the Georgetown University School of Medicine, Washington, DC. Dr. Khachemoune is from the Department of Dermatology, SUNY Downstate, Brooklyn, and the Department of Dermatology, Brooklyn Campus of the VA NY Harbor Healthcare System. 
livedoid lesions. ${ }^{3}$ Initial biopsies of these lesions pointed to viral exanthema and thrombotic vasculopathy as potential etiologies of morbilliform and livedoid lesions, respectively. Interestingly, patients may present with multiple cutaneous morphologies of the disease at the same time. ${ }^{3}$ The acral lesions ("COVID toes") have been popularized throughout the media and thus may be the best-known cutaneous manifestation of the disease at this time. New findings continuously arise, and further research is warranted as lesions that develop in hospitalized COVID-19 patients could be virus related or secondary to hospital-induced skin irritation, stressors, or medications. ${ }^{3}$ Importantly, clinicians should be aware of these cutaneous signs of COVID-19, especially when triaging patients.

\section{Risk Reduction}

The current health crisis could have a drastic impact on dermatology patients and providers. One factor that may increase COVID-19 risk in dermatology patients is immunosuppression. Many patients are on immunomodulators and biologics for skin conditions, which can cause immunosuppression directly and indirectly. Immunosuppression is a risk factor for severe disease in patients with COVID-19, so this population is at higher risk for serious infection. ${ }^{7}$ Telemedicine for nonemergent cases and follow-ups should be considered to decrease traffic in high-risk hospitals; to limit the number of people in waiting rooms; and to protect staff, providers, and patients alike. ${ }^{1}$ Recommendations for teledermatology consultation during this time include the following: First, have patients take photographs of their skin lesions and send them remotely to the consulting physician. If the lesion is easily recognizable, treatment recommendations can be made remotely; if the diagnosis is ambiguous, the dermatologist can set up an in-person appointment. ${ }^{1}$

\section{Personal Protective Equipment}

Moreover, the current need to wear personal protective equipment (PPE) and wash hands frequently may lead to skin disease among health care providers. Facial rashes may arise from wearing masks and goggles, and repeated handwashing and wearing gloves may lead to hand dermatitis. ${ }^{8}$ One study examined adverse skin reactions among health care workers $(\mathrm{N}=322)$ during the SARS outbreak in 2003. More than one-third (35.5\%) of staff members who wore masks regularly during the outbreak reported adverse skin reactions, including acne (59.6\%), facial itching $(51.4 \%)$, and rash $(35.8 \%){ }^{8}$ The acne etiology likely is multifactorial. Masks increase heat and humidity in the covered facial region, which can cause acne flare-ups due to increased sebum production and Cutibacterium acnes growth. ${ }^{8}$ Additionally, tight N95 masks may occlude the pilosebaceous glands, causing acne to flare. In the SARS study, facial itchiness and rashes likely were due to irritant contact dermatitis to the N95 masks. All of the respondents with adverse skin reactions from masks developed them after using N95 masks; those who wore surgical masks did not report reactions. ${ }^{8}$ Because $\mathrm{N} 95$ masks are recommended for health care workers caring for patients with highly transmissible respiratory infections such as SARS and COVID-19, it will be difficult to avoid wearing them during the current crisis. For this reason, topical retinoids and topical benzoyl peroxide should be the first-line treatment of mask-induced acne, and moisturization and topical corticosteroids should be used for facial erythema. Additionally, $21.4 \%$ of respondents reported adverse skin reactions from latex gloves during the SARS outbreak, including dry skin, itchiness, rash, and wheals. ${ }^{8}$ These skin reactions may have been type I IgE-mediated hypersensitivity reactions or irritant contact dermatitis due to latex sensitization and frequent handwashing. No respondents reported skin reactions to plastic gloves. ${ }^{8}$ For this reason, health care providers should consider wearing plastic gloves in lieu of or under latex gloves to prevent hand dermatitis during this time. Moisturization, barrier creams, and topical corticosteroids also can help treat hand dermatitis. Frequently changing PPE may help prevent skin disease among the frontline health care workers, ${ }^{8}$ which posed a problem at the beginning of the COVID-19 outbreak as there was a PPE shortage. With industry and individuals coming together to make and donate PPE, it is now more widely available for our frontline providers.

\section{Financial Impact}

Finally, the pandemic is having an immense financial impact on dermatology. ${ }^{9}$ At the onset of the outbreak, our role as health care providers was to help slow the spread of COVID-19; for this reason, most elective procedures were cancelled, and many outpatient clinics closed. Both elective procedures and outpatient visits are central to dermatology, so many dermatologists worked less or not at all during this time, leading to a loss of revenue. The goals of these measures were to reduce transmissibility of the disease, to prevent the health care system from being overwhelmed with critical COVID-19 cases, and to allocate resources to the frontline providers. ${ }^{9}$ Although these measures were beneficial for slowing the spread of disease, they were detrimental to some providers' and practices' financial stability. Many dermatology practices have begun to reopen with COVID-19 precautions in place. For example, practices are limiting the number of patients that can be in the office at one time, mandating temperature readings upon check-in, and requiring masks be worn throughout the entire visit. With continued recommendations for individuals to stay at home as much as possible, the number of patients being seen in dermatology clinics on a daily basis remains less than normal. One potential solution is telemedicine, which would allow patients' concerns to be addressed while keeping providers practicing with a normal patient volume during this time. ${ }^{9}$ Keeping providers financially afloat is vital for private practices to continue operating after the pandemic. Dermatology appointments are in high demand with long waiting lists during nonpandemic times; without dermatologists practicing at full capacity, 
DERMATOLOGIC IMPLICATIONS OF COVID-19

there will be an accumulation of patients with dermatologic conditions with even longer waiting times after the pandemic. Telemedicine may help reduce this potential accumulation of patients and allow patients to be treated in a more timely manner while alleviating financial pressure for providers.

Final Thoughts

The COVID-19 pandemic has spread across the world, infecting millions of people. Although the trends have slowed, more than 106,100 cases are still being diagnosed daily according to the Johns Hopkins University Coronavirus Resource Center (https://coronavirus.jhu.edu/map.html). Patients with COVID-19 may present with a variety of cutaneous lesions. Wearing PPE to take care of COVID-19 patients may lead to skin irritation, so care should be taken to address these adverse skin reactions to maintain the safety of providers. Finally, dermatologists should consider telemedicine during this time to protect high-risk patients, prevent a postpandemic surge of patients, and alleviate financial stressors caused by COVID-19.

REFERENCES

1. Tao J, Song Z, Yang L, et al. Emergency management for preventing and controlling nosocomial infection of 2019 novel coronavirus: implications for the dermatology department [published online March 5, 2020]. Br J Dermatol. doi:10.1111/bjd.19011.

2. Lippi G, Plebani M, Michael HB. Thrombocytopenia is associated with severe coronavirus disease 2019 (COVID-19) infections: a meta-analysis [published online March 13, 2020]. Chin Chit Acta. doi:10.1016/j.cca.2020.03.022.

3. Young S, Fernandez AP. Skin manifestations of COVID-19 [published online May 14, 2020]. Clove Chin J Med. doi:10.3949/ccjm.87a.ccc031.

4. Recalcati S. Cutaneous manifestations in COVID-19: a first perspective [published online March 26, 2020]. J Eur Acad Dermatol Venereal. doi:10.1111/jdv.16387.

5. Joob B, WiwanitkitV. COVID-19 can present with a rash and be mistaken for Dengue [published online March 22, 2020]. J Am Acad Dermatol. doi:10.1016/j.jaad.2020.03.036.

6. Casa s CG, Catalá A, Hernandez GC, et al. Classification of the cutaneous manifestations of COVID-19: a rapid prospective nationwide consensus study in Spain with 375 cases [published online April 29, 2020]. Br J Dermatol. doi:10.1111/bjd.19163.

7. Conforti C, Giuffrida R, Dianzani C, et al. COVID-19 and psoriasis: is it time to limited treatment with immunosuppressants? a call for action [published online March 11, 2020]. Dermatol Then. doi:10.1111/dth.13298.

8. Foo CC, Goon AT, Leow $\mathrm{YH}$, et al. Adverse skin reactions to personal protective equipment against severe respiratory syndrome- a descriptive study in Singapore. Contact Dermatitis. 2006;55:291-294.

9. Heymann WR. The profound dermatological manifestations of COVID-19 [published online March 18, 2020]. Dermatology World Insights and Inquiries. https://www.aad.org/dw/dw-insights-and -inquiries/2020-archive/march/dermatological-manifestations-covid-19. Accessed May 21, 2020.

308 I CUTIS ${ }^{\circledR}$

WWW.MDEDGE.COM/DERMATOLOGY

Copyright Cutis 2020. No part of this publication may be reproduced, stored, or transmitted without the prior written permission of the Publisher. 